\title{
Associations of Fat and Lean Body Mass with Circulating Amino Acids in Older Men and Women
}

\section{Mikkola, Tuija M}

2020-05

Mikkola , T M , Salonen , M K , Kajantie , E , Kautiainen , H \& Eriksson , J G 2020 , ' Associations of Fat and Lean Body Mass with Circulating Amino Acids in Older Men and Women ' , Journals of Gerontology. Series A: Biological Sciences and Medical Sciences , vol. 75 , no. 5 , pp. 885-891 . https://doi.org/10.1093/gerona/glz126

http://hdl.handle.net/10138/329709

https://doi.org/10.1093/gerona/glz126

acceptedVersion

Downloaded from Helda, University of Helsinki institutional repository.

This is an electronic reprint of the original article.

This reprint may differ from the original in pagination and typographic detail.

Please cite the original version. 


\title{
Associations of fat and lean body mass with circulating amino acids in
}

\author{
older men and women
}

Tuija M. Mikkola, ${ }^{1 *}$ Minna K. Salonen, ${ }^{1,2}$ Eero Kajantie, ${ }^{2,3,4,5}$ Hannu Kautiainen, ${ }^{1,6}$ Johan G. Eriksson $^{1,2,7,8,9}$

${ }^{1}$ Folkhälsan Research Center, Helsinki, Finland; ${ }^{2}$ Public Health Promotion Unit, National Institute for Health and Welfare, Helsinki, Finland; ${ }^{3}$ PEDEGO Research Unit, MRC Oulu, Oulu University Hospital and University of Oulu, Oulu, Finland; ${ }^{4}$ Department of Clinical and Molecular Medicine, Norwegian University for Science and Technology, Trondheim, Norway; ${ }^{5}$ Children's Hospital, Helsinki University Hospital and University of Helsinki, Helsinki, Finland; ${ }^{6}$ Primary Health Care Unit, Kuopio University Hospital, Finland; ${ }^{7}$ Department of General Practice and Primary Health Care, University of Helsinki and Helsinki University Hospital, Helsinki, Finland; ${ }^{8}$ Singapore Institute for Clinical Sciences, Agency for Science, Technology, and Research, Singapore; ${ }^{9}$ Department of Obstetrics \& Gynaecology, Yong Loo Lin School of Medicine, National University of Singapore, Singapore, Singapore

*Corresponding author: tuija.mikkola@folkhalsan.fi

Other email addresses: $\underline{\text { minna.salonen@thl.fi }}$, eero.kajantie@thl.fi, $\underline{\text { hannu.kautiainen@ medcare.fi }}$ johan.eriksson@helsinki.fi

(C) The Author(s) 2019. Published by Oxford University Press on behalf of The Gerontological Society of America. All rights reserved. For permissions, please e-mail: journals.permissions@oup.com. 
Abstract

Circulating amino acids are potential markers of body composition. Previous studies are mainly limited to middle age and focus on either fat or lean mass, thereby ignoring overall body composition. We investigated the associations of fat and lean body mass with circulating amino acids in older men and women.

We studied 594 women and 476 men from the Helsinki Birth Cohort Study (age 62-74 years). Bioelectrical impedance analysis was used to indicate two main body compartments by fat (fat mass/height ${ }^{2}$ ) and lean mass indices (lean mass/height ${ }^{2}$ ), dichotomized based on sex-specific medians. Eight serum amino acids were quantified using nuclear magnetic resonance spectroscopy. General linear models were adjusted for age, smoking and fasting glucose.

Higher lean mass index was associated with higher concentrations of branched-chain amino acids in both sexes $(\mathrm{p} \leq 0.001)$. In men, lean mass index was also positively associated with tyrosine ( $\mathrm{p}=0.006)$ and inversely with glycine $(\mathrm{p}<0.001)$. Higher fat mass index was associated with higher concentrations of all branched-chain amino acids, aromatic amino acids (phenylalanine and tyrosine), and alanine in both sexes $(\mathrm{p} \leq 0.008)$.

Associations between body composition and amino acids are largely similar in older men and women. The associations are largely similar to those previously observed in younger adults.

Key words: Metabolomics, Obesity, Muscles, Amino Acids 
Introduction

Human body composition undergoes substantial changes with age. With aging, lean body mass, largely composed of muscle mass, is progressively lost $(1,2)$. Concomitantly, the total amount of fat mass increases (1), and adipose tissue undergoes changes in distribution and function (3). Body composition has an important influence on physical functioning in older adults. Maintaining muscle mass is vital in order to manage daily activities and maintain mobility $(4,5)$. Low muscle mass may even increase mortality (6). Excess adipose tissue is also a risk factor for reduced physical capacity $(7,8)$ and functioning (9). The effect can be direct or indirect through elevated risks for e.g. osteoarthritis, type 2 diabetes, and cardiovascular diseases (3).

Metabolomics, i.e. determination of small particles in serum related to metabolism, have been applied in search for biomarkers for clinical conditions and in uncovering biological mechanisms contributing to non-communicable diseases and their risk factors. Concentrations of circulating amino acids, especially branched-chain amino acids (BCAAs), have been linked to obesity, insulin resistance, and type 2 diabetes in young and middle-aged adults (10-12). However, although body composition typically differs between older and younger adults, only few studies have investigated the relationships between body composition and amino acids in older adults. Most of the prior studies in older adults have had small sample sizes or focused on selective groups of older adults, such as functionally-limited older adults $(13,14)$. These studies have suggested that higher levels of circulating BCAAs and aromatic amino acids (AAAs) are associated with obesity/adiposity but also with lean body mass $(13,15-19)$. Prior studies have also focused on either fat mass or lean body mass and have not taken into account the remaining component of the overall body. This may be problematic as the amount of lean and fat mass are closely coupled; individuals with high fat mass 
also tend to have high lean mass. Hence, associations found between amino acids and fat or lean mass may partly be confounded by the remaining component of the body. Consequently, it could be that part of the associations reported between fat mass or BMI and amino acids are, in fact, due to lean mass. Therefore, it is critical to study these associations by taking into account both main body compartments simultaneously. Furthermore, to the best of our knowledge, associations between amino acids and body composition have not been reported separately in older men and older women despite the fact that men and women differ in their body composition. Better knowledge on the associations between body composition and circulating amino acids could be a first step in identification of the cellular pathways, which lead to low muscle mass or high fat mass.

The purpose of this study was to examine the associations of lean and fat mass with serum amino acid concentrations separately in older men and women. We controlled for mutual confounding by fat and lean mass by using a 2x2 matrix derived from dichotomized fat and lean mass indices.

Methods

The Helsinki Birth Cohort Study (HBCS) consists of 13,345 singletons, who were born in Helsinki in 1934-1944 and who were still alive in 1971, when all residents of Finland received a unique personal identification number. A total of 8760 individuals were born in the Helsinki University Central Hospital, and of these people, 2902 individuals were randomly selected to participate in a clinical examination in 2001-2004 to reach a target of 2000 participants. In total, 2003 individuals participated in the clinical study. Follow-up examinations were performed in $2007(n=1083)$ at the age of 62 to 74 years (mean 66 SD 2.8 years) and in 2011-13 at a mean age 71 years. The study design and assessments during the clinical visits have been described in details previously (20-22). 
The study was approved by the Ethics Committee of Epidemiology and Public Health of the Hospital District of Helsinki and Uusimaa and that of the National Public Health Institute, Helsinki. The present analysis uses the data from the 2007 follow-up examinations and includes participants with data on body composition ( $\mathrm{n}=1070$ in total, 594 women, 476 men) and metabolomics. All measurements were performed by trained study nurses. Blood pressure was measured from the right arm while the subject was in a sitting position and was recorded as the mean of 2 successive readings from a mercury sphygmomanometer. Information on smoking was obtained from questionnaires administered in 2001- 04 and in 2011-13. Those who reported being current smokers either in 200104 or in 2011-13 were considered smokers, others were considered non-smokers.

\section{Body composition and anthropometry}

Height was measured, without shoes on, to the nearest $0.1 \mathrm{~cm}$, and weight was measured in light indoor clothing to the nearest $0.1 \mathrm{~kg}$. Body mass index (BMI) was calculated as weight in kilograms divided by the square of height in meters. Body composition was assessed by bioelectrical impedance analysis using the InBody 3.0 eight-polar tactile electrode system (Biospace Co, Ltd, Seoul, Korea) (23). The instrument estimates lean body mass and body fat mass by segmental multifrequency $(5,50,250$, and $500 \mathrm{kHz})$ analyses. The measurements were made with the subject standing in light clothing on the 4-foot electrodes on the platform of the analyzer and gripping the two palm and thumb electrodes. Fat and lean mass indices were calculated: fat mass index $(\mathrm{FMI})=$ fat mass/height $[\text { in meters }]^{2}$ and lean mass index $(\mathrm{LMI})=$ lean mass/height $[\text { in meters }]^{2}$. Fat and lean mass indices were divided according to median values, separately for men and women, after which four body composition categories were created: 1) low FMI and low LMI 2) low FMI and high LMI 3) high FMI and low LMI 4) high FMI and LMI. By dividing participants into these categories, we aimed at reducing confounding between fat and lean mass indices. Supplemental 
Figure 1 shows the relationships between lean mass index and fat mass index and the median-split categories of body composition in women and men.

\section{Metabolomics}

Blood samples were drawn after overnight fasting. A high-throughput nuclear magnetic resonance metabolomics platform was applied for the quantification of lipid and abundant metabolite measures from serum samples in the HBCS during the 2007 clinical visit. The methods that were applied have been described in greater detail previously $(12,24)$. In this study, we focused on amino acids, namely alanine, glycine, histidine, phenylalanine, tyrosine, leucine, isoleucine, and valine. Sum scores were created for glucogenic (alanine and glycine), aromatic (histidine, phenylalanine, and tyrosine), and branched-chain (leucine, isoleucine, and valine) amino acids by computing z-scores for each amino acid and summing them up. Fasting plasma glucose was analyzed using a hexokinase method.

\section{Statistical analysis}

Relationships between fat mass index and lean mass index were derived from linear regression models. Differences in amino acid profiles between the sexes were determined using multivariate approach by using bootstrap type multivariate Hotelling T-squared test; it is a method to compare means of all variables of interest simultaneously (in the present analysis the amino acid profiles) while maintaining the chosen magnitude of Type I error. The relationships of body composition categories with background characteristics and amino acids were analyzed using general linear models. The main and interactive effects of fat and lean mass indices were analyzed by entering dichotomized lean mass index and fat mass index variables and their interaction as independent variables into the models. Age, fasting glucose concentration and smoking were introduced into the 
models as covariates when appropriate. In the case of violation of the assumptions (e.g. nonnormality), a bootstrap-type test was used. The normality of variables was evaluated by the ShapiroWilk W test. Cohen's effect-size statistics (d) was used as an indicator of strength of body compositions' effects on amino acids. Cohen's standard for effect size values above $0.20,0.50$ and 0.80 represent small, moderate and large effect sizes, respectively (25). Partial correlations (adjusted for age, fasting glucose concentration, and smoking) between percent body fat and amino acids were calculated with Sidak-adjusted (multiplicity) probabilities. All analyses were performed using STATA 15.0 (StataCorp, College Station, TX, USA).

Results

Characteristics of study participants are presented in Table 1 (women) and Table 2 (men). Women had higher mean fat mass index than men (9.4 SD 3.6 vs. 6.1 SD 2.4, t-test $\mathrm{p}<0.001)$ but lower mean lean mass index than men (17.8 SD 1.6 vs. 20.6 SD 1.7, p<0.001). The proportions of smokers among women were 16\% (low FMI low LMI), 18\% (low FMI high LMI), 29\% (high FMI low LMI), and 24\% (high FMI high LMI) (FMI p=0.022, LMI p=0.78, interaction $\mathrm{p}=0.40$ ) and among men 25\% (low FMI low LMI), 34\% (low FMI high LMI), 20\% (high FMI low LMI), and 20\% (high FMI high LMI) $($ FMI $\mathrm{p}=0.029, \mathrm{LMI} \mathrm{p}=0.42$, interaction $\mathrm{p}=0.31)$, respectively. The amino acid profiles differed between men and women $(\mathrm{p}<0.001)$ (Supplemental Table 1). Men had higher concentrations of histidine, tyrosine, leucine, isoleucine, and valine than women but lower glycine concentrations. Alanine or phenylalanine concentrations did not differ between men and women. 
There were no interactions between fat and lean mass on amino acid scores (Fig. 1). Of the individual amino acids, only valine was affected by the interaction of fat and lean mass index in women ( $\mathrm{p}=0.049)$ (Fig. 2).

Glucogenic amino acid score, adjusted for age, smoking, and fasting glucose concentration, was associated with fat mass index in women (Cohen's $d=0.26$, 95\% CI 0.10 to 0.42 , $\mathrm{p}=0.017$ ) (Fig. 1). Of the glucogenic amino acids, alanine was positively associated with fat mass index in both women $(\mathrm{d}=0.40,95 \%$ CI 0.24 to $0.56, \mathrm{p}<0.001)($ Fig. 2$)$ and men $(\mathrm{d}=0.39,95 \%$ CI 0.21 to $0.57, \mathrm{p}<0.001)$ (Fig. 3). Glycine, in turn, was inversely associated with lean mass in men (d=0.40, 95\% CI 0.22 to 0.59, p $<0.001$ ) (Fig. 3). Aromatic amino acid score was positively associated with fat mass index in women $(\mathrm{d}=0.54,95 \%$ CI 0.37 to $0.70, \mathrm{p}=0.006)$ and men $(\mathrm{d}=0.59,95 \% \mathrm{CI}, 0.41$ to $0.78, \mathrm{p}<0.001)$ (Fig.1). Of the individual AAAs, phenylalanine and tyrosine were associated with fat mass index in women (phenylalanine $\mathrm{d}=0.64,95 \%$ CI 0.48 to $0.81, \mathrm{p}<0.001$; tyrosine $\mathrm{d}=0.48,95 \%$ CI 0.32 to 0.64 , $\mathrm{p}<0.001$ ) and men (phenylalanine $\mathrm{d}=0.67,95 \%$ CI 0.49 to $0.86, \mathrm{p}<0.001$; tyrosine $\mathrm{d}=0.56,95 \%$ CI 0.37 to 0.74 ) (Fig. 2 and 3), and tyrosine was positively associated with lean mass index in men (d=0.45, 95\% CI 0.27 to $0.63, \mathrm{p}=0.003$ ) (Fig. 3). Branched-chain amino acid score was positively associated with both fat and lean mass indices in both women (FMI d= $0.67,95 \%$ CI 0.50 to 0.83 , $\mathrm{p}<0.001 ; \mathrm{LMI} d=0.60,95 \%$ CI 0.44 to $0.76, \mathrm{p}<0.001$ ) and men (FMI d=0.55, 95\% CI 0.37 to 0.74 , $\mathrm{p}<0.001$; LMI d=0.54, 95\% CI 0.36 to 0.72, p<0.001) (Fig. 1). All individual BCAAs (leucine, isoleucine, valine) were positively associated with lean and fat mass indices in both sexes $(\mathrm{p}<0.008)$ (Fig. 2 and 3). The strength of effect (d) between individual BCAAs and fat mass index was 0.45 (95\% CI 0.29 to 0.62 ) for leucine, 0.51 (95\% CI 0.34 to 0.67 ) for isoleucine, and 0.54 (95\% CI 0.38 to 0.70 ) for valine in women. In men, the corresponding figures were 0.54 (95\% CI 0.36 to 0.73 ) for leucine, 0.61 (95\% CI 0.43 to 0.80 ) for isoleucine, and 0.38 (95\% CI 0.20 to 0.56 ) for valine. The strength of effect between individual BCAAs and lean mass index was 0.43 (95\% CI 0.27 to 0.60 ) 
for leucine, 0.44 (95\% CI 0.28 to 0.61 ) for isoleucine, and 0.47 (95\% CI 0.31 to 0.63 ) for valine in women. In men, the corresponding figures were 0.51 (95\% CI 0.33 to 0.69 ) for leucine, 0.53 (95\% CI 0.35 to 0.71$)$ for isoleucine, and 0.45 (95\% CI 0.26 to 0.63 ) for valine.

Correlations of percent body fat with amino acids and amino acid scores are shown in Supplemental Table 2.

Discussion

This study investigated the associations of body composition with serum amino acid concentrations among older men and women. As fat and lean mass are correlated, we controlled for the mutual confounding by fat and lean mass by using a $2 \times 2$ matrix in categorizing the subjects. The associations were largely similar in men and women. Higher fat mass was associated with higher aromatic and branched-chain amino acid scores in both sexes and also with higher glucogenic amino acid score in older women. Higher lean body mass was associated with higher branched-chain amino acid score in both sexes. A novel finding was an inverse association between lean body mass and glycine among men.

The findings on the associations between lean body mass and serum branched-chain amino acids are in line with those of previous studies. Jourdan and colleagues reported that fat free mass index, measured using bioelectrical impedance analysis, was positively associated with the sum of BCAA concentrations in men and women aged 54-75 years (15). Another study in women, with a mean age of 47 years, reported that BCAA concentrations were positively associated with absolute lean mass 
and lean mass index, determined using dual energy x-ray absorptiometry (DXA) (17). BCAAs were also associated with fat free mass index and thigh muscle cross-sectional area in a small sample of functionally limited older men and women (13) and with appendicular lean mass index (lean mass of the lower limbs $/ \mathrm{h}^{2}$ ) in older black men (16). Positive associations between indicators of lean mass and serum AAA concentrations have also been reported in older adults $(15,16)$. In the present study, higher levels of tyrosine, an aromatic amino acid, were associated with higher lean mass index but only in men.

Possible mechanisms linking circulating branched-chain amino acid concentrations and lean mass include the stimulating effect of BCAAs on protein synthesis in muscle tissue (26). BCAAs can stimulate protein synthesis through mTOR signaling pathway (26). Of the BCAAs, leucine is particularly effective in upregulating protein synthesis $(27,28)$. It has been suggested that leucine intake is particularly important for older adults in preventing muscle loss (29).

Glycine, a glucogenic amino acid, was inversely associated with lean mass in men. To the best of our knowledge, associations between glycine and lean mass have not previously been reported in older adults. Men had lower glycine levels than women in the present study, which is in line with sex differences reported in prior studies $(12,30)$. Glycine has also been found to be lower in those with higher BMI (30). Further, glycine levels have been shown to increase after bariatric surgery in younger adults $(31,32)$. However, it is unclear whether this increase in glycine level is due to loss of fat mass, loss of lean mass or some other metabolic change following substantial weight loss. In the light of our results, the rise in glycine levels accompanying weight loss might be related to loss of lean mass, which typically occurs with fat loss (33). The association between lean mass and glycine is also supported by prior findings, which link sarcopenia, i.e. reduced muscle mass and function, 
with mitochondrial dysfunction and the glycine pathway. It has been hypothesized that sarcopenia has its origin in mitochondrial dysfunction (34). Mitochondrial dysfunction, in turn, has been reported to lead to upregulation of serine/1-carbon/glycine pathway in skeletal muscle of mice (35). This upregulation of serine/1-carbon/glycine pathway is plausibly a part of a compensatory response against increased mitochondrial stress to preserve cellular function (36). Hence, it could be speculated that rising serum glycine levels might serve as a marker of sarcopenia among older men, but more research is needed to confirm this.

Fat mass index was positively associated with AAAs, namely with tyrosine and phenylalanine, and BCAAs in both men and women. These associations were largely independent of those with lean mass index. In line with our findings, higher body mass index was associated with higher levels of tyrosine and phenylalanine and BCAAs in the Framingham Offspring cohort studied at a mean age of 55 years (18). Further, a study on 2400 women aged $40-80$ years reported positive associations between BCAAs and visceral fat mass determined using DXA (19). Our findings are also supported by dietary weight-loss interventions, in which weight loss was directly related to the decline of circulating BCAAs, tyrosine, and phenylalanine (37).

Many studies in younger adults have linked elevated BCAA levels to obesity, insulin resistance and type 2 diabetes $(10,38,39)$. Obesity and disturbances in glucose metabolism are intertwined as obesity is an important risk factor for insulin resistance and type 2 diabetes. Hence, disturbances in glucose metabolism in a part of the subjects with high adiposity in our cohort might explain some of the association observed between fat mass and BCAAs. Some studies reported an amino acid score including tyrosine, phenylalanine, and isoleucine to strongly predict future diabetes (38) and cardiovascular disease (40). BCAAs have been suggested to be indicators of metabolic disturbances 
rather than that of obesity $(10,41)$. BCAA overload may even have a causal role in developing insulin resistance and T2DM (10,29). It has been suggested that high BCAA levels, when combined with low IGF-1, activate the mTOR/S6K1 kinase pathway and phosphorylation of IRS1 on multiple serines, leading to insulin resistance (10). According to another theory, individuals with impaired BCAA metabolism could develop $\beta$-cell mitochondrial dysfunction through accumulation of toxic BCAA metabolites (11). On the other hand, BCAAs could be just a side product of the process of worsening metabolism or related to a possible compensatory mechanism in response to obesity $(11,29)$. Nevertheless, we observed associations between fat mass and BCAAs regardless of adjusting the present analyses for fasting glucose concentration.

The levels of BCAAs may also be affected by diet (42). Vegetarian and vegan diets have been found to be associated with lower levels of BCAAs, and higher levels of BCAAs may indicate an obesitogenic dietary pattern containing high amounts of animal and milk proteins (43). Further, a weight-loss intervention study suggested that the decline in AA concentrations during weight loss varies according to the protein content of the diet (37). However, some researchers have argued that BCAA intake is only weakly associated with fasting BCAA levels (44).

The present findings also suggest that concentrations of alanine, a glucogenic amino acid, are higher in those with high fat mass. Alanine was also found to be associated with BMI in a sample consisting of younger and older adults (18). Alanine is an important substrate and regulator for glucose metabolism. Decline in circulating alanine has been frequently reported after weight-loss interventions (45). Alanine is also related to BCAA catabolic pathway (46). Hence, elevated alanine levels can simply reflect the higher BCAA catabolic activity in individuals with high fat mass. 
A strength in this study is that we took into account both fat and lean mass, which may have mutual confounding effect on amino acids. Furthermore, we had a relatively large community sample including both men and women. We also analyzed several (groups of) amino acids. This study has also some limitations. The cross-sectional design limits drawing conclusions on causality. Compared to dual-energy x-ray absorptiometry, the accuracy of bioelectrical impedance analysis in determining fat and lean mass is more limited (47). We had no information on insulin resistance of the participants, which could have helped in dissecting the roles of adiposity and glucose/insulin metabolism in more detail.

\section{Conclusions}

The present study suggests that the associations between body composition and serum amino acids are largely similar in older community-dwelling men and older women. Both lean and fat mass are positively associated with branched-chain amino acids. In addition, fat mass is associated with aromatic amino acid concentrations. Despite marked differences in body composition between younger and older adults, the present findings suggest that the circulating amino acid profile is largely similarly linked to obesity in older adults than in younger adults. The present study identified potential candidate molecules to be focused on in future studies investigating cellular pathways leading to low lean mass or high fat mass in older adults. Especially, the role of glycine in development of sarcopenia and low muscle mass needs to be focused upon.

\section{Acknowledgements}

Author contributions: TMM participated in the design of the study, analysis and interpretation of the data and drafted the manuscript. MKS participated in the interpretation of the data and revised the draft critically for important intellectual content. EK participated in the conception and design of the 
study, interpretation of the data and revised the draft critically for important intellectual content. HK analyzed the data, participated in the interpretation of the results and revised the draft critically for important intellectual content. JGE participated in the conception and design of the study, collection of the data, interpretation of the data and revised the draft critically for important intellectual content. All authors accepted the final version of the manuscript.

Sponsor's role: The sponsor had no role in study design; in the collection, analysis and interpretation of data; in the writing of the report; or in the decision to submit the article for publication.

\section{Conflict of Interest}

None.

\section{Funding}

This work was supported by Emil Aaltonen Foundation; Finnish Foundation for Diabetes Research;

Foundation for Pediatric Research, Novo Nordisk Foundation; Signe and Ane Gyllenberg Foundation; Sigrid Jusélius Foundation; Samfundet Folkhälsan; Finska Läkaresällskapet; Liv och Hälsa; European Commission within the 7th Framework Programme (DORIAN, grant agreement no. 278603); and European Union Horizon 2020 programme (LifeCycle grant no.733206, DYNAHEALTH grant no. 633595 and RECAP grant no. SC1-2016-RTD-733180). The Academy of Finland supported EK (grant no. 127437, 129306, 130326, 134791, 263924 and 274794); and J.G.E. (grant no. 129369, 129907, 135072, 129255, and 126775). 


\section{References}

1. Kyle UG, Genton L, Hans D, Karsegard L, Slosman DO, Pichard C. Age-related differences in fat-free mass, skeletal muscle, body cell mass and fat mass between 18 and 94 years. Eur $J$ Clin Nutr. 2001;55(8):663-672.

2. Goodpaster BH, Study for the HABC, Park SW, et al. The Loss of Skeletal Muscle Strength, Mass, and Quality in Older Adults: The Health, Aging and Body Composition Study. $J$ Gerontol A Biol Sci Med Sci. 2006;61(10):1059-1064.

3. Tchkonia T, Morbeck DE, Von Zglinicki T, et al. Fat tissue, aging, and cellular senescence. Aging Cell. 2010;9(5):667-684.

4. Hairi NN, Cumming RG, Naganathan V, et al. Loss of muscle strength, mass (sarcopenia), and quality (specific force) and its relationship with functional limitation and physical disability: The concord health and ageing in men project. J Am Geriatr Soc. 2010;58(11):2055-2062.

5. Visser M, Goodpaster BH, Kritchevsky SB, et al. Muscle Mass, Muscle Strength, and Muscle Fat Infiltration as Predictors of Incident Mobility Limitations in Well-Functioning Older Persons. J Gerontol A Biol Sci Med Sci. 2005;60(3):324-333.

6. Genton L, Graf CE, Karsegard VL, Kyle UG, Pichard C. Low fat-free mass as a marker of mortality in community-dwelling healthy elderly subjects. Age Ageing. 2013;42(1):33-39.

7. Mikkola TM, von Bonsdorff MB, Salonen MK, et al. Body composition as a predictor of physical performance in older age: a ten-year follow-up of the Helsinki Birth Cohort Study. Arch Gerontol Geriatr. 2018;77(May):163-168.

8. Jerome GJ, Ko S-U, Chiles Shaffer NS, Studenski SA, Ferrucci L, Simonsick EM. CrossSectional and Longitudinal Associations Between Adiposity and Walking Endurance in 
Adults Age 60-79. J Gerontol A Biol Sci Med Sci. 2016;71(12):1661-1666.

9. Beavers KM, Miller ME, Rejeski WJ, Nicklas BJ, Krichevsky SB. Fat mass loss predicts gain in physical function with intentional weight loss in older adults. J Gerontol A Biol Sci Med Sci. 2013;68(1):80-86.

10. Newgard CB, An J, Bain JR, et al. A Branched-Chain Amino Acid-Related Metabolic Signature that Differentiates Obese and Lean Humans and Contributes to Insulin Resistance. Cell Metab. 2009;9(4):311-326.

11. Lynch CJ, Adams SH. Branched-chain amino acids in metabolic signalling and insulin resistance. Nat Rev Endocrinol. 2014;10(12):723-736.

12. Wurtz P, Soininen P, Kangas AJ, et al. Branched-chain and aromatic amino acids are predictors of insulin resistance in young adults. Diabetes Care. 2013;36(3):648-655.

13. Lustgarten MS, Price LL, Chale A, Phillips EM, Fielding RA. Branched Chain Amino acids are associated with muscle mass in functionally limited older adults. J Gerontol A Biol Sci Med Sci. 2014;69(6):717-724.

14. Lustgarten MS, Lyn Price L, Phillips EM, Fielding RA. Serum glycine is associated with regional body fat and insulin resistance in functionally-limited older adults. PLoS One. $2013 ; 8(12): 8-14$

15. Jourdan C, Petersen AK, Gieger C, et al. Body fat free mass is associated with the serum metabolite profile in a population-based study. PLoS One. 2012;7(6).

16. Murphy RA, Moore SC, Playdon M, et al. Metabolites Associated with Lean Mass and Adiposity in Older Black Men. J Gerontol A Biol Sci Med Sci. 2017;72(10):1352-1359.

17. Korostishevsky M, Steves CJ, Malkin I, Spector T, Williams FMK, Livshits G. Genomics and metabolomics of muscular mass in a community-based sample of UK females. Eur J Hum 
Genet. 2016;24(2):277-283.

18. Ho JE, Larson MG, Ghorbani A, et al. Metabolomic profiles of body mass index in the Framingham Heart Study reveal distinct cardiometabolic phenotypes. PLoS One. 2016;11(2):1-16.

19. Menni C, Migaud M, Glastonbury CA, et al. Metabolomic profiling to dissect the role of visceral fat in cardiometabolic health. Obesity. 2016;24(6):1380-1388.

20. Barker DJP, Osmond C, Forsén TJ, Kajantie E, Eriksson JG. Trajectories of Growth among Children Who Have Coronary Events as Adults. N Engl J Med. 2005;353(17):1802-1809.

21. Ylihärsilä H, Kajantie E, Osmond C, Forsén T, Barker DJ, Eriksson JG. Body mass index during childhood and adult body composition in men and women aged 56-70 y. Am J Clin Nutr. 2008;87(6):1769-1775.

22. Eriksson JG, Osmond C, Perälä M-MM, et al. Prenatal and childhood growth and physical performance in old age - findings from the Helsinki Birth Cohort Study 1934-1944. Age (Omaha). 2015;37(6):108.

23. Malavolti M, Mussi C, Poli M, et al. Cross-calibration of eight-polar bioelectrical impedance analysis versus dual-energy X-ray absorptiometry for the assessment of total and appendicular body composition in healthy subjects aged 21-82 years. Ann Hum Biol. 2003;30(4):380-391.

24. Soininen P, Kangas AJ, Würtz P, et al. High-throughput serum NMR metabonomics for costeffective holistic studies on systemic metabolism. Analyst. 2009;134(9):1781-1785.

25. Cohen J. Statistical Power Analysis for the Behavioral Sciences. 2nd ed. Lawrence Erlbaum; 1988.

26. Nie C, He T, Zhang W, Zhang G, Ma X. Branched chain amino acids: Beyond nutrition metabolism. Int J Mol Sci. 2018;19(4). 
27. Crozier SJ, Kimball SR, Emmert SW, Anthony JC, Jefferson LS. Oral leucine administration stimulates protein synthesis in rat skeletal muscle. J Nutr. 2005;135(3):376-382.

28. Churchward-Venne TA, Breen L, Di Donato DM, et al. Leucine supplementation of a lowprotein mixed macronutrient beverage enhances myofibrillar protein synthesis in young men: A double-blind, randomized trial1-3. Am J Clin Nutr. 2014;99(2):276-286.

29. Manders RJ, Little JP, Forbes SC, Candow DG. Insulinotropic and muscle protein synthetic effects of branched-chain amino acids: Potential therapy for type 2 diabetes and sarcopenia. Nutrients. 2012;4(11):1664-1678.

30. Kraus WE, Pieper CF, Huffman KM, et al. Association of Plasma Small-Molecule Intermediate Metabolites with Age and Body Mass Index Across Six Diverse Study Populations. J Gerontol A Biol Sci Med Sci. 2016;71(11):1507-1513.

31. Tan HC, Khoo CM, Tan MZ-W, et al. The Effects of Sleeve Gastrectomy and Gastric Bypass on Branched-Chain Amino Acid Metabolism 1 Year After Bariatric Surgery. Obes Surg. 2016;26(8):1830-1835.

32. Gralka E, Luchinat C, Tenori L, Ernst B, Thurnheer M, Schultes B. Metabolomic fingerprint of severe obesity is dynamically affected by bariatric surgery in a procedure-dependent manner. Am J Clin Nutr. 2015;102(6):1313-1322.

33. Heymsfield SB, Gonzalez MCC, Shen W, Redman L, Thomas D. Weight Loss Composition is One-Fourth Fat-Free Mass. A Critical Review and Critique. Obes Rev. 2015;15(4):310-321.

34. Marzetti E, Calvani R, Cesari M, et al. Mitochondrial dysfunction and sarcopenia of aging: From signaling pathways to clinical trials. Int J Biochem Cell Biol. 2013;45(10):2288-2301.

35. Ost M, Keipert S, Van Schothorst EM, et al. Muscle mitohormesis promotes cellular survival via serine/glycine pathway flux. FASEB J. 2015;29(4):1314-1328. 
36. Koopman R, Caldow MK, Ham DJ, Lynch GS. Glycine metabolism in skeletal muscle: Implications for metabolic homeostasis. Curr Opin Clin Nutr Metab Care. 2017;20(4):237242.

37. Zheng Y, Ceglarek U, Huang T, et al. Weight-loss diets and 2-y changes in circulating amino acids in 2 randomized intervention trials. Am J Clin Nutr. 2016;103(2):505-511.

38. Wang TJ, Larson MG, Vasan RS, et al. Metabolite Profiles and the Risk of Developing Diabetes. Nat Med. 2011;17(4):448-453.

39. Guillet C, Masgrau A, Walrand S, Boirie Y. Impaired protein metabolism: Interlinks between obesity, insulin resistance and inflammation. Obes Rev. 2012;13(SUPPL.2):51-57.

40. Magnusson M, Lewis GD, Ericson U, et al. A diabetes-predictive amino acid score and future cardiovascular disease. Eur Heart J. 2013;34(26):1982-1989.

41. Batch BC, Shah SH, Newgard CB, et al. Branched chain amino acids are novel biomarkers for discrimination of metabolic wellness. Metabolism. 2013;62(7):961-969.

42. Duggan GE, Hittel DS, Hughey CC, Weljie A, Vogel HJ, Shearer J. Differentiating short- and long-term effects of diet in the obese mouse using $1 \mathrm{H}$-nuclear magnetic resonance metabolomics. Diabetes, Obes Metab. 2011;13:859-862.

43. Elshorbagy A, Jernerén F, Basta M, Basta C. Amino acid changes during transition to a vegan diet supplemented with fish in healthy humans. Eur J Nutr. 2017:1953-1962.

44. Shah SH, Crosslin DR, Haynes CS, et al. Branched-chain amino acid levels are associated with improvement in insulin resistance with weight loss. Diabetologia. 2012;55(2):321-330.

45. Tulipani S, Griffin J, Palau-Rodriguez M, et al. Metabolomics-guided insights on bariatric surgery versus behavioral interventions for weight loss. Obesity. 2016;24(12):2451-2466. 
46. Xie B, Waters MJ, Schirra HJ. Investigating potential mechanisms of obesity by metabolomics. J Biomed Biotechnol. 2012;2012.

47. Achamrah N, Colange G, Delay J, et al. Comparison of body composition assessment by DXA and BIA according to the body mass index: A retrospective study on 3655 measures. PLoS One. 2018;13(7):e0200465. 
Table 1. Characteristics of women of the Helsinki Birth Cohort Study according to fat mass index (FMI) and lean mass index (LMI) in 2007.

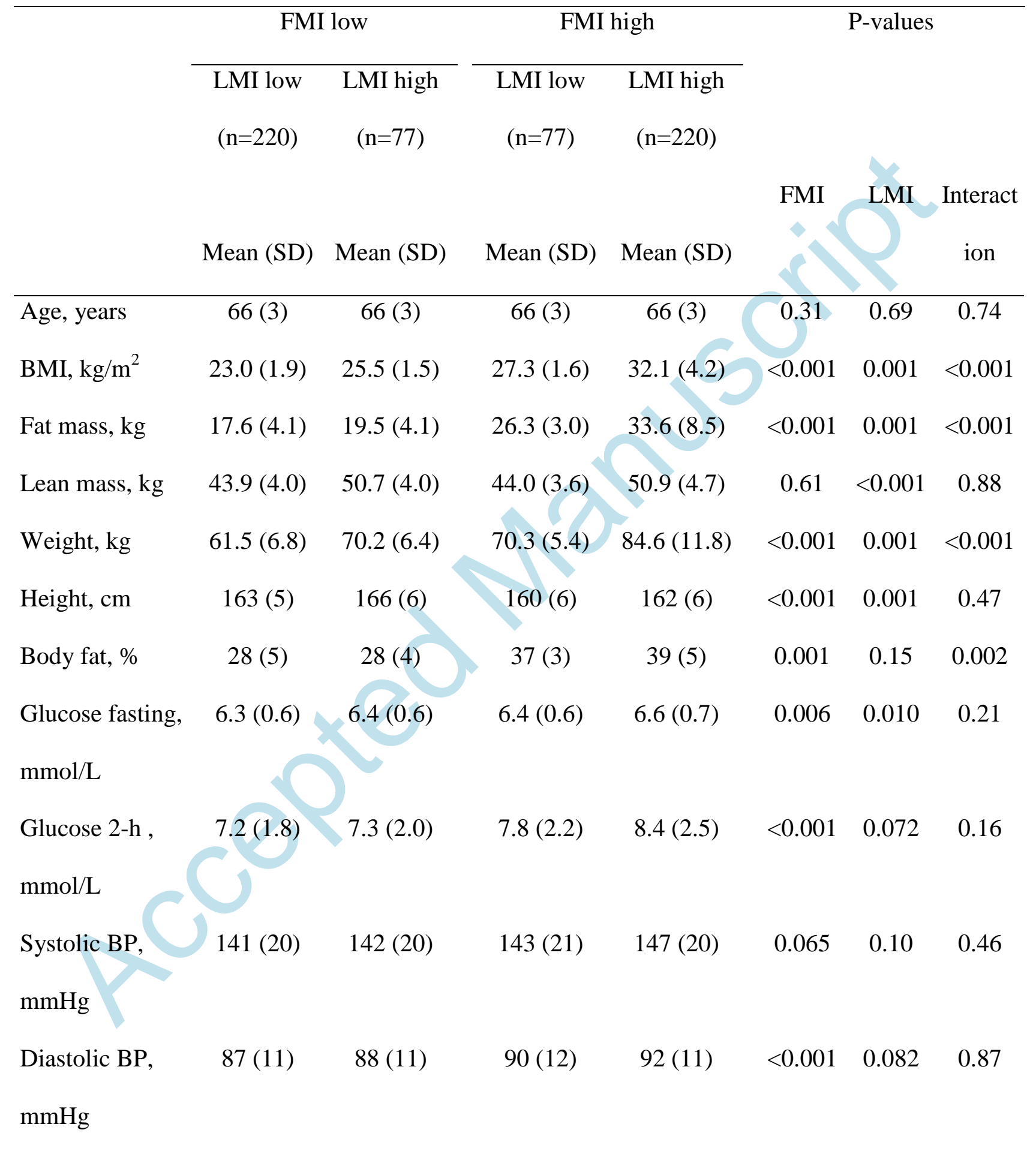

Note. BMI, body mass index; High lean mass index $>17.7 \mathrm{~kg} / \mathrm{m}^{2}$, low lean mass index $\leq 17.7$ $\mathrm{kg} / \mathrm{m}^{2}$, high fat mass index $>8.9 \mathrm{~kg} / \mathrm{m}^{2}$, low fat mass index $\leq 8.9 \mathrm{~kg} / \mathrm{m}^{2}$

Table 2. Characteristics of men of the Helsinki Birth Cohort Study according to fat mass index 
(FMI) and lean mass index (LMI) in 2007.

\begin{tabular}{|c|c|c|c|c|c|c|c|}
\hline & \multicolumn{2}{|c|}{ FMI low } & \multicolumn{2}{|c|}{ FMI high } & \multicolumn{3}{|c|}{ P-values } \\
\hline & $\begin{array}{l}\text { LMI low } \\
(n=164)\end{array}$ & $\begin{array}{l}\text { LMI high } \\
\quad(n=74)\end{array}$ & $\begin{array}{l}\text { LMI low } \\
(\mathrm{n}=74)\end{array}$ & $\begin{array}{l}\text { LMI high } \\
(\mathrm{n}=164)\end{array}$ & & & \\
\hline & Mean (SD) & Mean (SD) & Mean (SD) & Mean (SD) & FMI & LMI & Interaction \\
\hline Age, years & $65.4(2.2)$ & $65.6(2.6)$ & $65.7(2.9)$ & $66.4(2.9)$ & 0.11 & 0.062 & 0.35 \\
\hline BMI, $\mathrm{kg} / \mathrm{m} 2$ & $26.1(1.2)$ & $30.5(2.9)$ & $23.3(1.6)$ & $26.6(1.5)$ & $<0.001$ & $<0.001$ & 0.012 \\
\hline Fat mass, $\mathrm{kg}$ & $15.0(2.6)$ & $25.7(7)$ & $13.4(3.1)$ & $21.4(3.6)$ & $<0.001$ & $<0.001$ & 0.004 \\
\hline Lean mass, $\mathrm{kg}$ & $69.0(4.7)$ & $69.0(6.7)$ & $60.1(5.1)$ & $58.3(4.2)$ & 0.11 & $<0.001$ & 0.091 \\
\hline Weight, kg & $83.9(5.9)$ & $94.8(10.7)$ & $73.4(6.8)$ & $79.6(6.1)$ & $<0.001$ & $<0.001$ & 0.004 \\
\hline Height, $\mathrm{cm}$ & $179(5.1)$ & $176(6.2)$ & $177(5.7)$ & $173(5.4)$ & $<0.001$ & $<0.001$ & 0.18 \\
\hline Body fat, \% & $17.8(2.5)$ & $26.9(4.8)$ & $18.1(3.3)$ & $26.8(3.2)$ & $<0.001$ & 0.83 & 0.56 \\
\hline Glucose fasting, & $6.6(0.6)$ & $6.9(0.7)$ & $6.7(0.6)$ & $6.8(0.6)$ & 0.023 & 0.009 & 0.31 \\
\hline $\mathrm{mmol} / \mathrm{L}$ & & & & & & & \\
\hline $\begin{array}{l}\text { Glucose 2-h, } \\
\text { mmol/L }\end{array}$ & $7.3(2.0)$ & (2.4) & $8.6(2.5)$ & $8.2(2.6)$ & $<0.001$ & 0.40 & 0.57 \\
\hline Systolic blood & $143(21)$ & 144 (17) & $148(22)$ & $149(18)$ & 0.011 & 0.62 & 0.93 \\
\hline $\begin{array}{l}\text { Diastolic blood } \\
\text { pressure, mmHg }\end{array}$ & $88(11)$ & $88(10)$ & $91(12)$ & $93(10)$ & $<0.001$ & 0.40 & 0.30 \\
\hline
\end{tabular}

Note. BMI, body mass index; High lean mass index $>20.5 \mathrm{~kg} / \mathrm{m}^{2}$, low lean mass index $\leq 20.5$ $\mathrm{kg} / \mathrm{m}^{2}$, high fat mass index $>5.7 \mathrm{~kg} / \mathrm{m}^{2}$, low fat mass index $\leq 5.7 \mathrm{~kg} / \mathrm{m}^{2}$ 
Figure Legends

Fig 1. Adjusted (age, fasting glucose and smoking) amino acid scores across body composition categories in women and men. 'LMI low' refers to lean mass index (lean body mass $/ \mathrm{h}^{2}$ ) equal or below the sex-specific median and 'LMI high' to lean mass index above the sex-specific median. 'FMI low' and 'FMI high' are defined correspondingly. Amino acid scores are calculated by summing up the z-scores of the respective amino acid concentrations.

Fig. 2 Adjusted (age, fasting glucose and smoking) amino acid concentrations across body composition categories in women. 'LMI low' refers to lean mass index (lean body mass $/ \mathrm{h}^{2}$ ) equal or below the sex-specific median and 'LMI high' to lean mass index above the sex-specific median. 'FMI low' and 'FMI high' are defined correspondingly.

Fig. 3 Adjusted (age, fasting glucose and smoking) amino acid concentrations across body composition categories in men. 'LMI low' refers to lean mass index (lean body mass $/ \mathrm{h}^{2}$ ) equal or below the sex-specific median and 'LMI high' to lean mass index above the sex-specific median. 'FMI low' and 'FMI high' are defined correspondingly. 


\section{Figure 1}
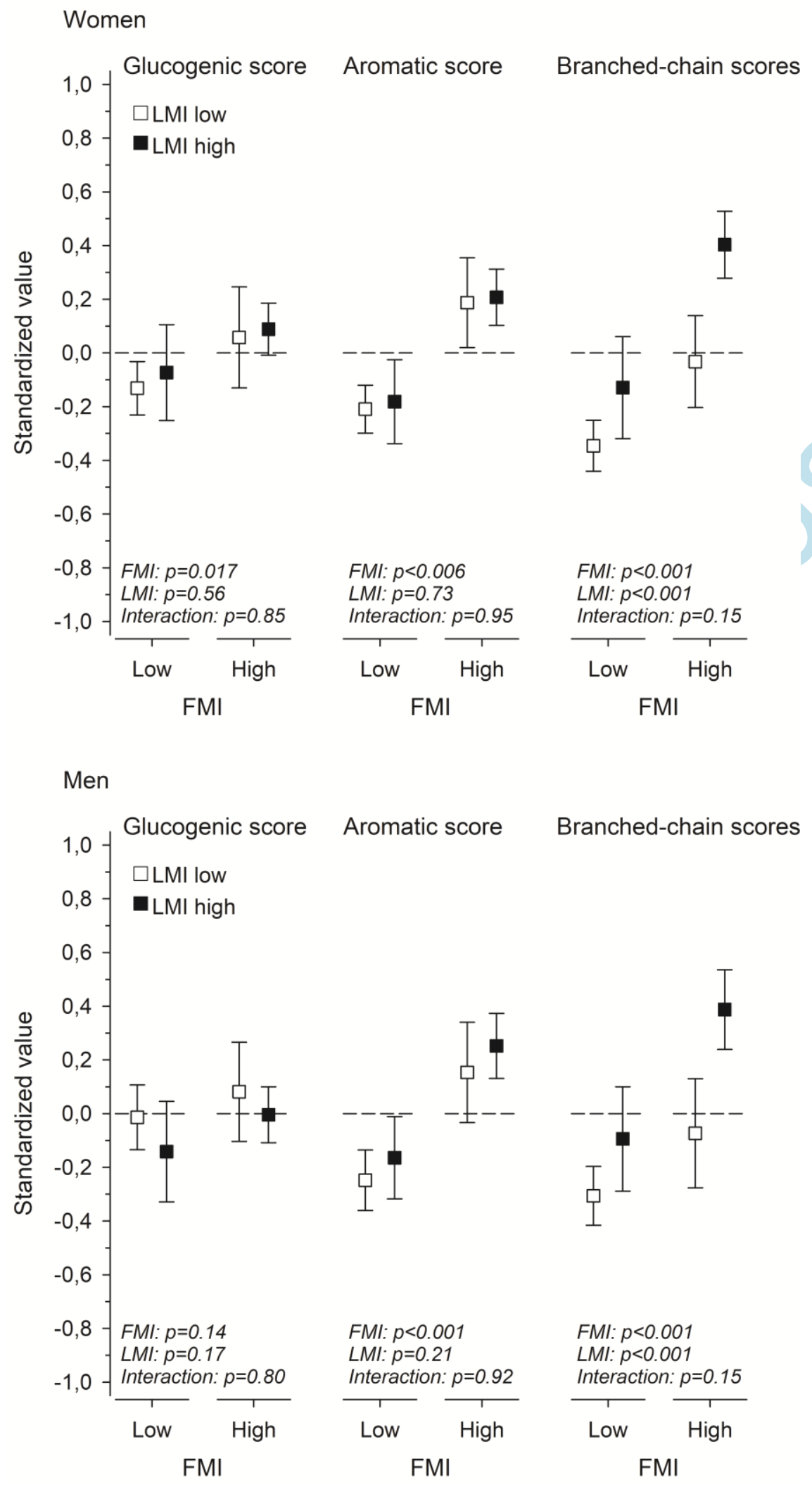
Figure 2

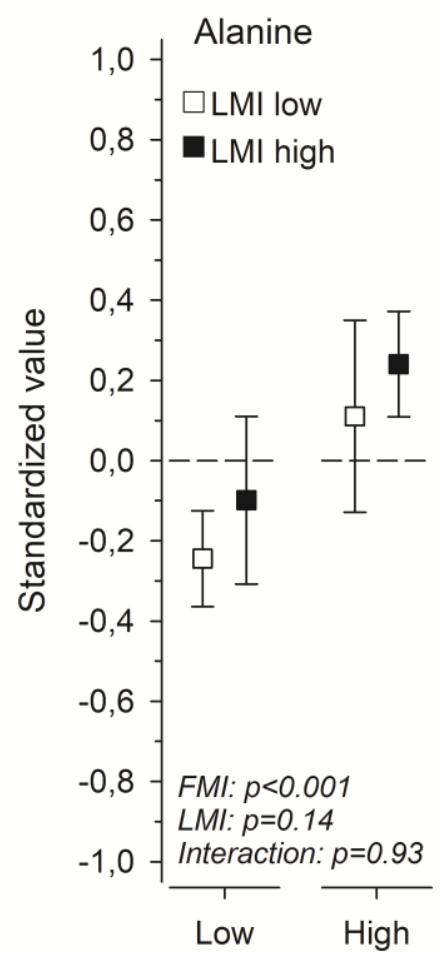

FMI
Glycine
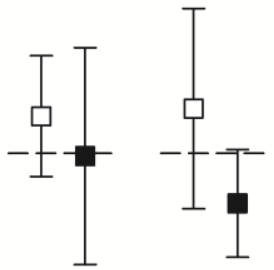

FMI: $p=0.64$

LMI: $p=0.12$

Interaction: $p=0.52$

Low

FMI

Leucine

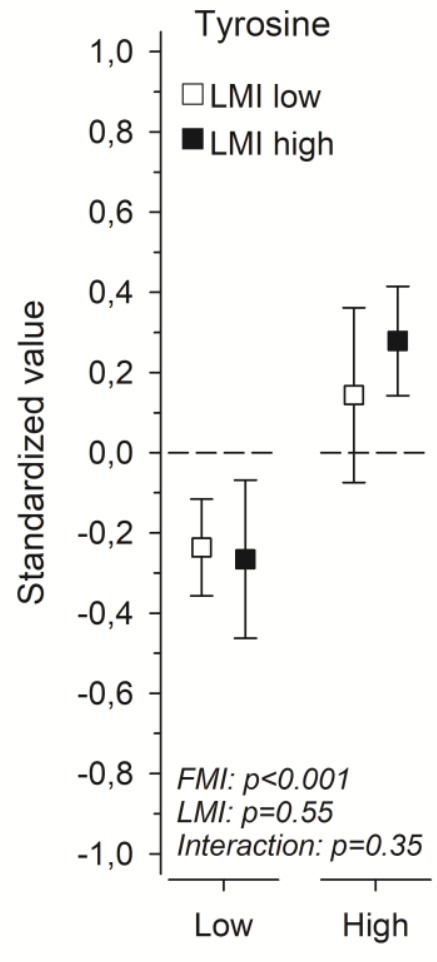

FMI
Histidine

Phenylalanine

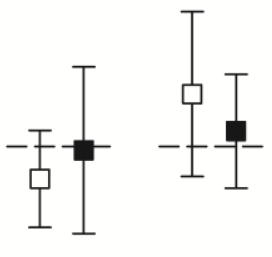

FMI: $p=0.15$

LMI: $p=0.91$

Interaction: $p=0.35$

Low High

FMI

Isoleucine

Valine
FMI: $p<0.001$

LMI: $p=0.74$

Interaction: $p=0.89$

Low High

FMI 
Figure 3

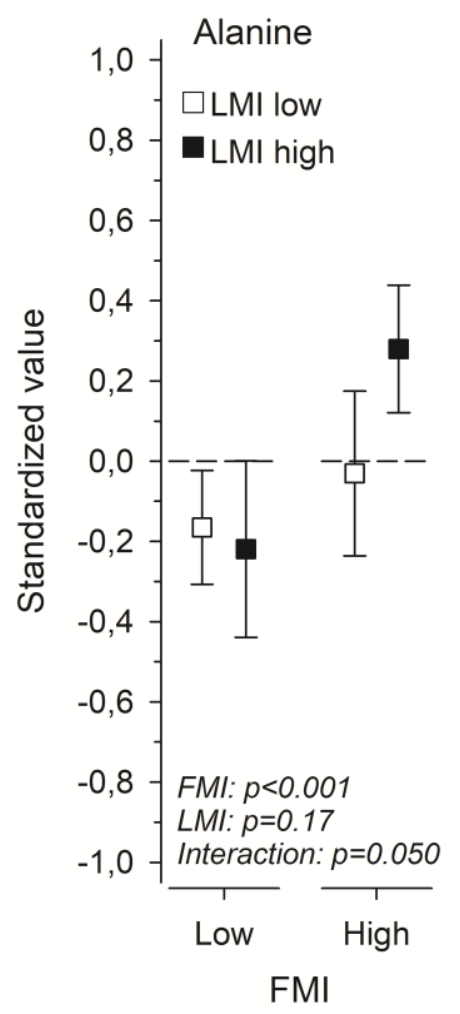

Glycine

Histidine

Phenylalanine
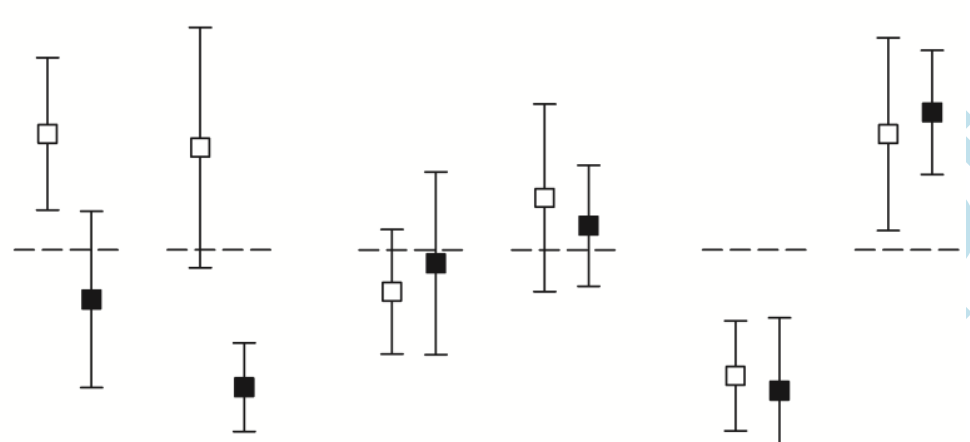

FMI: $p=0.25$

LMI: $p<0.001$

Interaction: $p=0.40$

FMI: $p=0.11$

LMI: $p=0.99$

Interaction: $p=0.48$

0.001

LMI: $p=0.93$

Low

FMI

Low High

FMI

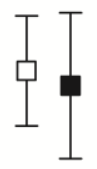

Interaction: $p=0.63$

Low High

FMI

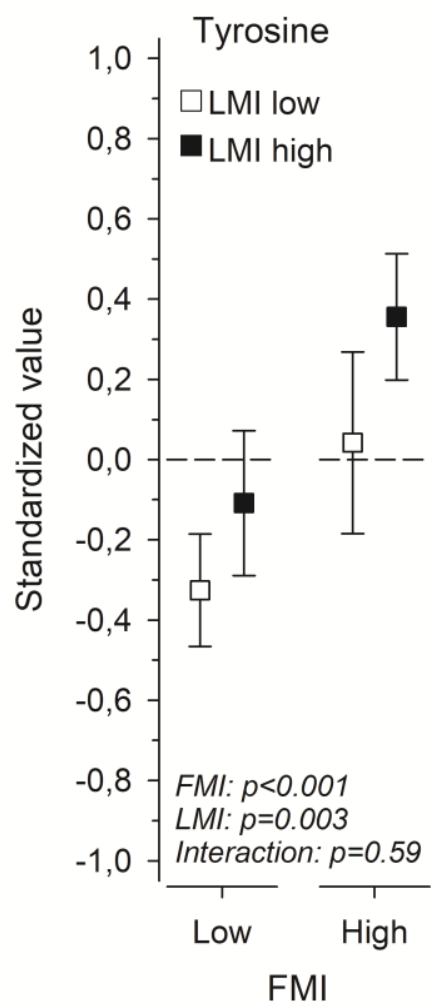

Leucine

Isoleucine

Valine
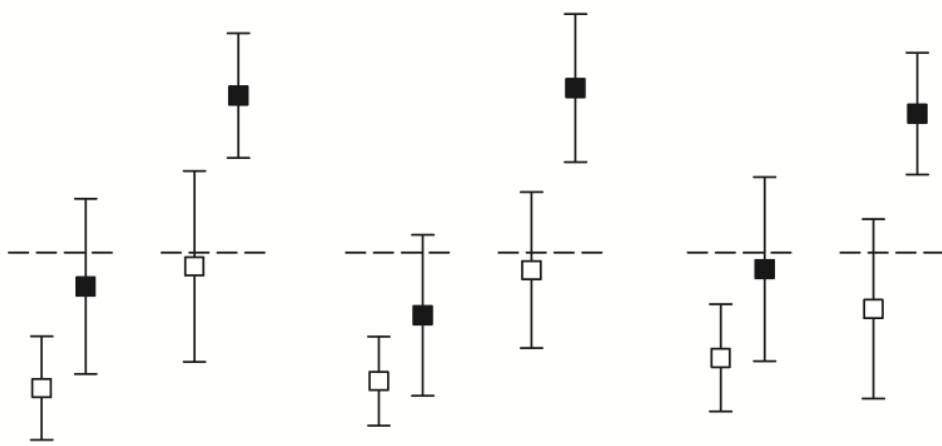

FMI: $p<0.001$

LMI: $p<0.001$

FMI: $p<0.001$

LMI: $p<0.001$

$p=0.007$

Interaction: $p=0.38$

Interaction: $p=0.11$

LMI: $p<0.001$

Low $\frac{1}{\text { High }}$

FMI

Low High

FMI 